QUARTERLY OF APPLIED MATHEMATICS

VOLUME LXVIII, NUMBER 3

SEPTEMBER 2010, PAGES 557-561

S 0033-569X(2010)01209-1

Article electronically published on May 27, 2010

\title{
A LOCAL GREEDY ALGORITHM AND HIGHER-ORDER EXTENSIONS FOR GLOBAL NUMERICAL CONTINUATION OF ANALYTICALLY VARYING SUBSPACES
}

\author{
BY
}

\author{
KEVIN ZUMBRUN
}

Department of Mathematics, 223 Rawles Hall, Indiana University, Bloomington, Indiana 47405

\begin{abstract}
We present a family of numerical implementations of Kato's ODE propagating global bases of analytically varying invariant subspaces of which the first-order version is a surprisingly simple "greedy algorithm" that is both stable and easy to program and the second-order version a relaxation of a first-order scheme of Brin and Zumbrun. The method has application to numerical Evans function computations used to assess stability of traveling-wave solutions of time-evolutionary PDE.
\end{abstract}

1. Introduction. Let $P(\lambda) \in \mathbb{C}^{n \times n}$ be a projection, $P^{2}=P$, and subspace $S(\lambda) \subset$ $\mathbb{C}^{n}$ its range, with $P$ depending analytically on $\lambda$ within a simply connected subset $\Lambda$ of the complex plane. Then, a standard result in matrix perturbation theory $[\mathrm{K}$ is that there exists a global analytic basis $\left\{r_{j}(\lambda)\right\}$ of $S$ on $\Lambda$; moreover, expressing $r_{j}$ as column vectors, this can be prescribed constructively as the solution $R=\left(r_{1}, \ldots, r_{k}\right)$ of Kato's $O D E$

$$
R^{\prime}=\left(P^{\prime} P-P P^{\prime}\right) R, \quad R\left(\lambda_{0}\right)=R^{0},
$$

where the initializing value $R^{0}$ is any matrix whose columns form a basis for $S\left(\lambda_{0}\right)$, and "' denotes differentiation with respect to $\lambda$. This prescription is also "minimal" in the sense that $P R^{\prime} \equiv 0$, i.e., the derivative of basis $R$ lies entirely in the direction complementary to its span (the kernel of $P$ ); see [HSZ, HLZ] for further discussion.

The problem of computing such an analytically varying basis is important in numerical Evans function computations for determining stability of traveling waves. Roughly speaking, analytic bases for stable and unstable manifolds of certain limiting coefficient matrices are used to define an analytic Evans function, whose winding number around a contour $\Gamma \subset \Lambda$ counts the number of unstable eigenvalues enclosed by $\Gamma$ of the linearized

Received December 1, 2008.

2000 Mathematics Subject Classification. Primary 65L99.

This research was partially supported under NSF grants number DMS-0300487, DMS-0505780, and DMS-0801745.

E-mail address: kzumbrun@indiana.edu

(C)2010 Brown University 
operator about the wave, with zero winding number corresponding to stability. For further discussion, see [GZ, Br, BrZ, BDG, HSZ, HuZ, BHRZ, HLZ, CHNZ, HLyZ1, HLyZ2, and references therein.

Various algorithms for numerical determination of bases $R$ have been introduced in BrZ, BDG, HSZ, each of which turns out to be equivalent to (a discretization of) (1.1), and each of which is $O\left(n^{3}\right)$ in complexity (though with different coefficients). The purpose of this brief note is to introduce a new and particularly simple discretization of (1.1), which at the first order of accuracy consists of what might be called a "local greedy algorithm". Namely, choosing a set of mesh points $\lambda_{j}$ around $\Gamma$ and denoting by $R_{j}$ the approximation of $R\left(\lambda_{j}\right)$, our first-order scheme is simply

$$
R_{j+1}=P_{j+1} R_{j}, \quad R_{0}=R^{0} .
$$

That is, the continuation of basis $R$ to each new step is obtained by projecting the value at the previous step onto the new subspace: the simplest possible choice, and one that at first sight seems entirely local. However, remarkably, this local choice leads to a globally defined basis; in particular, upon traversing the entire contour $\Gamma$ and returning to $\lambda_{L}=\lambda_{0}$, we find that, up to convergence error, the value of $R_{L}$ returns to the starting value $R_{0}=R^{0}$. This is both simpler and faster than its closest relative in [BrZ]; indeed, it is completely trivial to program (the main issue in most Evans function applications).

This simplification is based on the reduced version

$$
R^{\prime}=P^{\prime} R, \quad R\left(\lambda_{0}\right)=R^{0},
$$

of (1.1) ([K], pp. 99-101), which readily yields minimal difference schemes to all orders of accuracy. A particularly attractive version when speed is an issue appears to be the second-order version, which is a relaxation of the first-order scheme in [BrZ].

1.1. The reduced ODE. We begin by recalling the properties of the reduced Kato ODE (1.3).

Proposition 1.1. There exists a global solution $R$ of (1.3) on any simply connected domain $\Lambda$ containing $\lambda_{0}$, with $\operatorname{rank} R(\lambda) \equiv \operatorname{rank} R^{0}$. Moverover, if $P\left(\lambda_{0}\right) R^{0}=R^{0}$, then: (i) $P R \equiv R$. (ii) $P R^{\prime} \equiv 0$. (iii) $R$ satisfies (1.1).

Proof. As a linear ODE with analytic coefficients, (1.3) possesses an analytic solution in a neighborhood of $\lambda_{0}$ that may be extended globally along any curve, whence, by the principle of analytic continuation, it possesses a global analytic solution on any simply connected domain containing $\lambda_{0}[\mathrm{~K}$. Constancy of $\operatorname{rank} R(\lambda)$ follows likewise by the fact that $R$ satisfies a linear ODE.

Differentiating the identity $P^{2}=P$ following $\left[\underline{\mathrm{K}}\right.$ yields $P P^{\prime}+P^{\prime} P=P^{\prime}$, whence, multiplying on the right by $P$, we find the key property

$$
P P^{\prime} P=0 .
$$

From (1.4), we obtain

$$
(P R-R)^{\prime}=\left(P^{\prime} R+P R^{\prime}-R^{\prime}\right)=P^{\prime} R+(P-I) P^{\prime} R=P P^{\prime} R,
$$

which, by $P P^{\prime} P=0$ and $P^{2}=P$, gives

$$
(P R-R)^{\prime}=-P P^{\prime}(P R-R), \quad(P R-R)\left(\lambda_{0}\right)=0,
$$


from which (i) follows by uniqueness of solutions of linear ODE. Expanding $P R^{\prime}=P P^{\prime} R$ and using $P R=R$ and $P P^{\prime} P=0$, we obtain $P R^{\prime}=P P^{\prime} P R=0$, verifying (ii). Finally, using (i) and (ii), we obtain $R^{\prime}=P^{\prime} R=P^{\prime} P R-P R^{\prime}=\left(P^{\prime} P-P P^{\prime}\right) R$, verifying (iii).

Remark 1.2. Conversely, (i)-(ii) imply (1.3) through $R^{\prime}=(P R)^{\prime}=P^{\prime} R+P R^{\prime}=$ $P^{\prime} R$.

1.2. Numerical implementation.

1.2.1. First-order version. Approximating $P^{\prime}\left(\lambda_{j}\right)$ to first order by the finite difference $\left(P_{j+1}-P_{j}\right) /\left(\lambda_{j+1}-\lambda_{j}\right)$ and substituting this into a first-order Euler scheme gives

$$
R_{j+1}=R_{j}+\left(\lambda_{j+1}-\lambda_{j}\right) \frac{P_{j+1}-P_{j}}{\lambda_{j+1}-\lambda_{j}} R_{j}
$$

or $R_{j+1}=R_{j}+P_{j+1} R_{j}-P_{j} R_{j}$, yielding greedy algorithm (1.2) by the property $P_{j} R_{j}=$ $R_{j}$ (Note: preserved exactly by the scheme).

REMARK 1.3. The same procedure applied to the original equation (1.1) yields

$$
R_{j+1}=R_{j}+\left(P_{j+1} P_{j}-P_{j} P_{j+1}\right) R_{j}
$$

or, following with a projection $P_{j+1}$ to stabilize the scheme without changing the order of accuracy, the first-order scheme

$$
R_{j+1}=P_{j+1} R_{j}+P_{j+1}\left(P_{j+1} P_{j}-P_{j} P_{j+1}\right) R_{j}=P_{j+1}\left(I+P_{j}\left(I-P_{j+1}\right)\right) R_{j} .
$$

introduced in $[\mathrm{BrZ}$. This is slightly more costly at two evaluations of $P$ on the average and three matrix multiplications vs. one evaluation of $P$ and one matrix multiplication for (1.2). Depending on the cost of evaluating $P$ and whether the mesh is fixed (in which case evaluations of $P$ may be shared), this can vary between approximately one and three times the cost of (1.2).

REMARK 1.4. An advantage of (1.2) is that it works even for merely continuously varying $P$. Thus, this algorithm extends well to the boundary $\partial \Lambda$ in the case (as for Evans analysis of multi-dimensional shocks [HLyZ2]) that it contains branch singularities or worse, something like a lower-order stiff ODE solver designed for stability rather than accuracy.

1.2.2. Second-order version. To obtain a second-order discretization of (1.3), we make the second-order approximation $R_{j+1}-R_{j} \approx \Delta \lambda_{j} P_{j+1 / 2}^{\prime} R_{j+1 / 2}$ with respect to $\Delta \lambda_{j}:=$ $\lambda_{j+1}-\lambda_{j}$. Noting that $R_{j+1 / 2} \approx P_{j+1 / 2} R_{j}$ to second order, by (1.2), and approximating $P_{j+1 / 2} \approx \frac{1}{2}\left(P_{j+1}+P_{j}\right)$, also good to second order, and $P_{j+1 / 2}^{\prime} \approx\left(P_{j+1}-P_{j}\right) / \Delta \lambda_{j}$, we obtain, putting everything together and rearranging,

$$
R_{j+1}=R_{j}+\frac{1}{2}\left(P_{j+1}-P_{j}\right)\left(P_{j+1}+P_{j}\right) R_{j} .
$$

Stabilizing by following with a projection $P_{j+1}$, we obtain after some rearrangement the reduced second-order explicit scheme

$$
R_{j+1}=P_{j+1}\left[I+\frac{1}{2} P_{j}\left(I-P_{j+1}\right)\right] R_{j},
$$

which may be recognized as a relaxation of the first-order scheme (1.5).

This has the same computational cost per step as (1.5), i.e., two evaluations of $P_{j}$ and three matrix multiplications, while the number of steps goes as $1 / \sqrt{\text { Tolerance }}$ vs. 
$1 /$ Tolerance for the first-order scheme (1.5), so is 10 times fewer for the typical value Tolerance $=10^{-2}$. For this typical tolerance value, therefore, there is a computational savings of ten times for (1.6) over (1.5). In the worst case that $P$ is inexpensive compared to matrix multiplication so that (1.6) is three times as expensive per step as (1.2), there is a computation savings of four times for (1.6) over (1.2). Thus, the second-order scheme (1.6) is the version we recommend for serious computations. For individual numerical experiments the simpler greedy algorithm (1.2) will often suffice (see discussion, Section 1.4).

1.3. Third and higher-order versions. Arbitrarily higher-order schemes may be obtained by Richardson extrapolation starting from scheme (1.2) or (1.6). For example, second-order Richardson extrapolation applied to (1.2) yields an alternative second-order scheme

$$
R_{j+1}=P_{j+1}\left(2 P_{j+1 / 2}-I\right) R_{j},
$$

while third-order extrapolation applied to (1.7) yields the third-order scheme

$$
R_{j+1}=P_{j+1}\left[\frac{4}{3}\left(2 P_{j+3 / 4}-I\right) P_{j+1 / 2}\left(2 P_{j+1 / 4}-I\right)-\frac{1}{3}\left(2 P_{j+1 / 2}-I\right)\right] R_{j} .
$$

Third-order extrapolation applied to (1.6) yields the simpler third-order scheme

$$
\begin{aligned}
R_{j+1}= & P_{j+1}\left[\frac{4}{3}\left(I+\frac{1}{2} P_{j+1 / 2}\left(I-P_{j+1}\right)\right) P_{j+1 / 2}\left(I+\frac{1}{2} P_{j}\left(I-P_{j+1 / 2}\right)\right)\right. \\
& \left.-\frac{1}{3}\left(I+\frac{1}{2} P_{j}\left(I-P_{j+1}\right)\right)\right] R_{j} .
\end{aligned}
$$

Here, fractional indices denote points along the line segment between $\lambda_{j}$ and $\lambda_{j+1 / 2}$ with corresponding fractional distance.

More generally, denoting by $\mathcal{T}^{m, h}$ the matrix advancing $R_{j}$ to $R_{j+1}$ for an $m$ th order scheme with step $h \in \mathbb{C}$, we obtain by Richardson extrapolation an $(m+1)$ st order scheme

$$
\mathcal{T}_{j}^{m+1,2 h}=\frac{2^{m}}{2^{m}-1} \mathcal{T}_{j+1}^{m, h} \mathcal{T}_{j}^{m, h}-\frac{1}{2^{m}-1} \mathcal{T}_{j}^{m, 2 h} .
$$

When $P$ is costly, it appears preferable to use schemes involving evaluations at only integer steps in order to share evaluations of $P$ (of course, this assumes a fixed, or non-adaptive, mesh, which may or may not be desirable). For example, Lagrange interpolation of explicit $m$ th-order Euler approximating derivatives of $P$ at $\lambda_{j}$ yields an $(m+1)$-step scheme with integer steps. However, the complexity of the resulting schemes makes these unappealing in practice.

Among higher-order schemes, we thus suggest only (1.6), or for strict tolerance (1.9).

1.4. Implementation in numerical Evans function computations. For Evans computations, $P(\lambda)$ is the eigenprojection onto the stable (unstable) subspace of a given matrix $A(\lambda)$. Thus, it may be prescribed uniquely as

$$
P(\lambda)=R\left(L^{*} R\right)^{-1} L^{*},
$$

for any choice of right and left bases $R$ and $L$ (matrices whose columns consist of basis elements, as before).

Ordered Schur decomposition, an $O\left(n^{3}\right)$ operation supported as an automatic function in programming packages such as MATLAB, applied to $A$ and $A^{*}$, respectively, gives 
orthonormal right and left bases of the left and right stable subspaces of $A$, hence an optimally conditioned choice in (1.10). Thus, evaluation of $P$ is in practice straightforward to program. On the other hand, it is typically an expensive (i.e., large coefficient) $O\left(n^{3}\right)$ call involving Schur decomposition and several matrix multiplications, so that it is desirable to minimize the number of evaluations of $P$ in numerical continuation algorithms. For a fixed, i.e., non-adaptive, mesh (at least for (1.2), (1.6), or explicit Euler schemes that are evaluated at mesh points only), $P$ need be evaluated only once for every mesh point, so that higher-order schemes are clearly preferable in this application. On the other hand, evaluation of the Evans function, involving solution of a further variable-coefficient ODE initialized with $R$, costs far more than the computation of $R$, so that these details can be ignored in most computations with (relatively) little effect. Ordered Schur decomposition (MATLAB version) has been used with good results in HuZ, HLyZ2.

\section{REFERENCES}

[BHRZ] B. Barker, J. Humpherys, K. Rudd, and K. Zumbrun, Stability of viscous shocks in isentropic gas dynamics, Comm. Math. Phys. 281 (2008), no. 1, 231-249. MR2403609 (2009c:35286)

[BDG] T.J. Bridges, G. Derks, and G. Gottwald, Stability and instability of solitary waves of the fifthorder KdV equation: A numerical framework, Phys. D, 172(1-4):190-216, 2002. MR1946769 (2004i:37148)

[Br] L.Q. Brin, Numerical testing of the stability of viscous shock waves, Math. Comp., 70(235):1071-1088, 2001. MR 1710652 (2001j:65118)

[BrZ] L.Q. Brin and K. Zumbrun, Analytically varying eigenvectors and the stability of viscous shock waves, Mat. Contemp., 22:19-32, 2002, Seventh Workshop on Partial Differential Equations, Part I (Rio de Janeiro, 2001). MR.1965784 (2004c:15012)

[CHNZ] N. Costanzino, J. Humpherys, T. Nguyen, and K. Zumbrun, Spectral stability of noncharacteristic boundary layers of isentropic Navier-Stokes equations, Arch. Ration. Mech. Anal. 192 (2009), no. 3, 537-587. MR2505363

[GZ] R. Gardner and K. Zumbrun, The gap lemma and geometric criteria instability of viscous shock profiles, CPAM 51. 1998, 797-855. MR1617251 (99c:35152)

[HLZ] J. Humpherys, O. Lafitte, and K. Zumbrun, Stability of isentropic viscous shock profiles in the high-mach number limit, to appear in Comm. Math. Phys.

[HLyZ1] J. Humpherys, G. Lyng, and K. Zumbrun, Spectral stability of ideal-gas shock layers, Arch. Ration. Mech. Anal. 194 (2009), no. 3, 1029-1079.

[HLyZ2] J. Humpherys, G. Lyng, and K. Zumbrun, Multidimensional spectral stability of large-amplitude Navier-Stokes shocks, in preparation.

[HSZ] J. Humpherys, B. Sandstede, and K. Zumbrun, Efficient computation of analytic bases in Evans function analysis of large systems, Numer. Math. 103 (2006), no. 4, 631-642. MR2221065 (2007a:65028)

[HuZ] J. Humpherys and K. Zumbrun, An efficient shooting algorithm for Evans function calculations in large systems, Physica D, 220(2):116-126, 2006. MR2253406 (2007e:35006)

[K] T. Kato, Perturbation theory for linear operators. Springer-Verlag, Berlin, Heidelberg (1985). 